\title{
Cavities and the effective pressure between abrading clasts and the bedrock
}

\author{
R. C. A. Hindmarsh \\ British Antarctic Survey, Natural Environment Research Council, High Cross, Madingley Road, Cambridge CB3 OET, England
}

\begin{abstract}
The mechanical configuration of cavities associated with clasts is discussed, and how this affects the water pressure underneath the clast. The range of permissible water pressure is determined by flow parallel to the bed rather than by flow towards the bed. The actual pressure is controlled by hydraulic connections to areas away from the clast. The degree of cavitation provides an additional degree of freedom which adjusts to ensure that horizontal and vertical forces respect the Coulomb friction condition.
\end{abstract}

\section{INTRODUCTION}

A clast in contact with temperate glacier ice and subglacial bedrock will either move or remain stationary. If moving, it is a tool for glacial abrasion. Clast motion is driven by the drag of ice moving relative to the clast, but is resisted by the Coulomb friction of the clast with the bed. In this paper the conditions controlling this drag and resistance are considered. As has been done by all recent papers on the subject of glacial abrasion (e.g. Boulton, 1974; Hallet, 1979), the computation of the effective pressure of the contact between clast and bed is considered in some detail. The problem in constructing a theory of abrasion arises from the notion that there is a continuous film of water enveloping the clast. In order to generate clast-bed contact forces, one must provide a mechanism which permits a water-pressure difference to exist between the upper and lower surfaces of the clast. Force balance then requires there to be clast-bed contact stresses greater than those necessary to oppose the weight of the clast.

Generating scratches by simple experiments with two rocks in the field or laboratory is sufficient to convince that contact forces must be higher than those generated by the buoyant weight of the clast. Moreover, the presence of striations on glacially eroded bedforms would seem to require high clast-bed contact stresses. While the shape of these forms can be partly explained by the presence of indentors on the clasts (e.g. Iverson, 1991), the construction of an abrasion theory where clast-bed contact stresses arise from bedward flow (e.g. Hallet, 1979 leads to a prediction of contact forces, which, in regions where the bed is flat, are unlikely to be more than an order of magnitude greater than the buoyant weight of the clast (Hallet, 1979, fig. 2). Another example which opposes the high-water-pressure theory is the erosional effect of particles transported by rivers, where scratching of bare bedrock is less common, but where contact forces are no less than the buoyant weight of the particle.
If, on the other hand, one allows the water pressure beneath the clast to be rather less than the ice pressure, thus generating high clast-bed contact forces (Boulton, 1974), one needs to provide a mechanism by which this low water pressure between clast and bedrock can be maintained, a point emphasised by Hallet (1979). Such low pressures are easy to understand if the bed is permeable and in contact with a region of lower pressure, but where the bed is impermeable the problem of finding mechanisms which generate low water pressures underneath the clast becomes more difficult. One mechanism is the formation of cavities on the lee side of clasts, analyzed and rejected as stable configurations on grounds of mechanical instability by Boulton and others (1979), and discussed further by Hindmarsh (1985), the point being that cavities will cause sub-clast water pressures to be low, owing to the hydraulic connection between the cavity and the sub-clast area. Clasts with cavities have been frequently observed (Boulton and others, 1979), but these are in places where the moving clast experiences a changing stress field. The implication of this is that one cannot make inferences about the stability of the clasts. In this paper, we examine theoretically the formation of cavities contiguous to clasts and consider their effect on lodgement and abrasion.

\section{THE SOLO GLAST}

\subsection{Basic configuration}

The configurations we are considering are illustrated in Figure 1. The clast lies between the ice and the bed, and is surrounded by water or gas, and clast-clast interactions provide a negligible part of the total force balance. The ice is assumed to be at its pressure-melting point. In general, the lower face of the clast and the bed will not be conforming, and in the gaps there will be either water or 


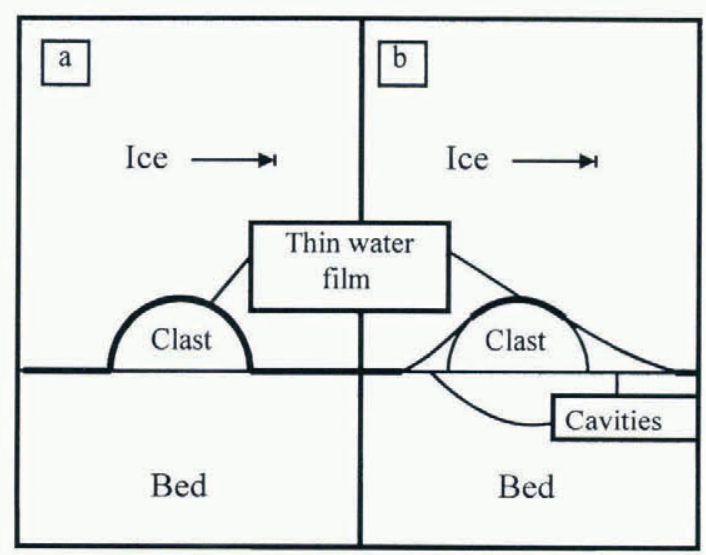

Fig. 1. Physical model of the clast used in this paper. The clast is enveloped in water or gas. The ice may be conforming with the clast virtually everywhere (a) or cavities may develop on both upstream and downstream sides (b). The cavities may be filled with water or with gas.

gas. The gas will either be air or water vapour, and in view of the stresses generated we can take the triple-point pressure of water to be zero.

By assumption, since the ice is nowhere in contact with the clast, the forces on the clast are not determined directly by stresses in the ice, but rather by how these stresses are transmitted directly through the fluids enveloping the clast. These assumptions are typical of those used in regelation theory and combinations of regelation theory and viscous-flow theory (e.g. Nye, 1969; $\mathrm{Kamb}, 1970$ ). The ice is disallowed to be in direct contact with the clast by the assumption of temperate conditions. This constraint is imposed by thermodynamic principles, which do not, however, disallow direct clast-bed contacts.

If one permits temporary, local freezing of ice onto the clast, as in the mechanism proposed by Robin (1976), then the "problem" of explaining high clast-bed contact pressures disappears, as there is no reason why water pressures under the clast should be similar to the stresses transmitted from ice directly to clast. Non-temperate conditions will not be considered further in this paper, but it should be borne in mind that they could potentially explain some abrasional episodes.

If the ice is separated from the upper surface of a clast by a thin water film but nevertheless conforming to all upper surfaces of the clast, continuity of pressure imposes constraints, and in particular it seems difficult to invoke arbitrary pressures under the clast to explain lodgement and abrasion. This is the key point made by Hallet (1979, 1981 ) in arguing against the Boulton (1974) model. We shall argue that there are mechanisms related to thinning of the water film which can seal the sub-clast area from the super-clast region, and that these areas necessarily exist, as the high hydraulic transmissibility of the sub-clast area requires very large fluxes of water to support the pressure gradients imposed by the flow of ice past a clast.

\subsection{Application of the Watts solution to the half-space}

The sub-clast water-pressure problem has been analyzed by Hallet $(1979,1981)$, who applied a theory due to
Watts (1974) describing the flow of "Newtonian" ice around a spherical clast. Hallet's configuration is shown in Figure 2. We shall call all solutions describing the flow of ice around clasts "Watts-type solutions".

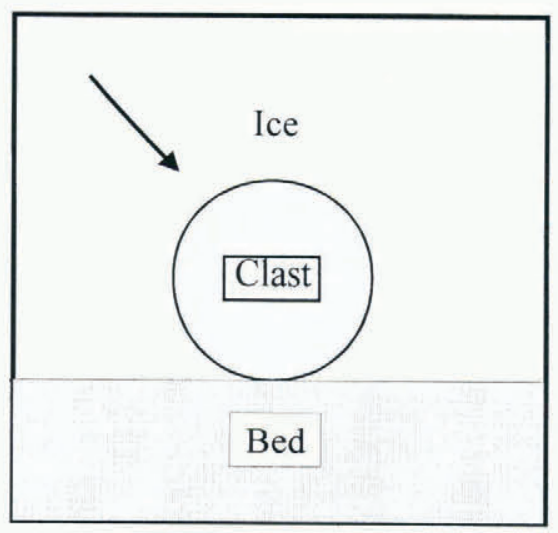

Fig. 2. The configuration used by Hallet (1979). The clast is surrounded by a thin water film.

Watts' solution is based on the following assumptions. The model of ice is one where all regelation occurs at the interface and where ice is a Newtonian fluid. In spherical co-ordinates $(r, \theta, \psi)$ the pressure is found to be

$$
p=-\frac{\mu U a^{3}}{r^{2}\left(a^{2}+a_{*}{ }^{2}\right)} \cos \theta
$$

and the normal stresses are

$$
\begin{aligned}
\sigma_{r r} & =3 \frac{a^{3} \mu U \cos \theta}{r^{2}\left(a^{2}+a_{*}{ }^{2}\right)} \\
\sigma_{\theta \theta} & =\sigma_{\varphi r}=0,
\end{aligned}
$$

where $U$ is the far-field velocity, $a$ is the radius of the sphere, and $a_{*}{ }^{2}=3 \mu C / L \rho_{\mathrm{s}}$ where $\mu$ is the viscosity of the ice, $C$ is the Clausius constant, $L$ is the volumetric latent heat of ice, and $\rho_{s}$ is the thermal resistivity of the system. The quantity $a_{*}$ represents a transition length scale - ice flows round clasts smaller than this by melting-refreezing processes and around clasts larger than this primarily by viscous deformation. The estimate by Watts that $a_{*}$ $\approx 10 \mathrm{~cm}$ is approximate. Fowler (1981) estimates the length scale as being $1 \mathrm{~cm}$. The Watts solution is linear and therefore does not include non-linear rheological effects or the formation of cavities.

From Cauchy's law we can see that $p_{\mathrm{w}}$ in the water film is equal to $\sigma_{r r}$, and using $x=a \cos \theta$ we can see that

$$
\sigma_{r r}(a)=3 \mu U x /\left(a^{2}+a_{*}{ }^{2}\right),
$$

i.e. there is a linear decline in the pressure in the water film from upstream end to downstream end. This is what we would also expect underneath the clast if Poiseuille flow were occurring there, and means that the net contact force between particle and bed would simply be the buoyant weight of the particle, as discussed by Hallet (1979).

Let us consider for the moment the velocity and stress fields within the ice and ignore the lubricating water film. In this case, the only geometry where the bed is close to the clast which is also consistent with the application of 
the Watts full-space theory is the half-space problem. This is because the shear stress is zero along the plane bisecting the domain in the Watts solution, and is also zero along this plane in the half-space assumption by virtue of the assumption of perfect slip.

However, when one considers the thin water film, applying Watts' model to the half-plane introduces inconsistencies (Hindmarsh, 1985). A pressure gradient beneath the clast immediately implies that this is an extra drainage route, which means that the Watts solution, with its assumption of restricted drainage around the clast, no longer applies. Recall that in classical regelation theory the thickness of the water film adjusts by the flow or melting of ice so as to provide the right flow resistance for the discharge and the water-pressure gradient. This is not possible underneath a clast, where the transmissibility is more or less constant and is almost certainly very much larger than that around the clast, because the same dependence on the cube of thickness occurs. As we suggested above, there is no particular reason why the clast base and the bed should be conforming.

A further problem with the direct application of the Watts theory lies in the fact that a water film exists between the ice and the bed upstream of the clast. This is a special property of the half-space solution. From Cauchy's law, the pressure in the water film between clast and ice at the leading edge of the clast is given by $\sigma_{r r}$, while that in the film between clast and bed at this point is given by $\sigma_{\theta \theta}$, and we can see from Equation (1) that these stresses are not equal, and the water pressure is discontinuous at $(r, \theta)=(a, j \pi), j=(0,1)$. This is illustrated in Figure 3. Application of the Watts theory to the half-space is not consistent, as it ignores the water film between bed and ice. More generally, one cannot have two free surfaces of a deforming fluid meeting at angles other than a right-angle, as shear stresses associated with the deformation are incompatible with a symmetric stress tensor in this case. It is not clear that the configuration discussed by Hallet (1979) is physically consistent. Of course, Hallet is simply using the Watts model as a physical example of what is essentially an order-of-magnitude argument, but in view of the comments made above about continuity of pressure in the water film one should be careful not to go outside the domain of applicability of this model.

These problems all stem from the requirement that the ice be conforming with the clast and the bed. We do not expect this to happen, and suggest that cavities will form, and that this resolves the problem of discontinuous water pressure in the thin film. There is another problem, that large water-pressure gradients imply large temperature gradients. We will ignore the thermodynamic problems, concentrating on a fluidmechanical resolution to the problems of consistency discussed above, and leave the thermodynamical considerations to a subsequent paper.

A final point relates to the longitudinal symmetry of the sphere. It is easy to see from the Watts solution that the total vertical force acting on both upper surface and lower surface is zero, and it would seem that this is a special consequence of the upstream/downstream symmetry of the solution. This fact need not be true for asymmetric configurations, and upwards or downwards

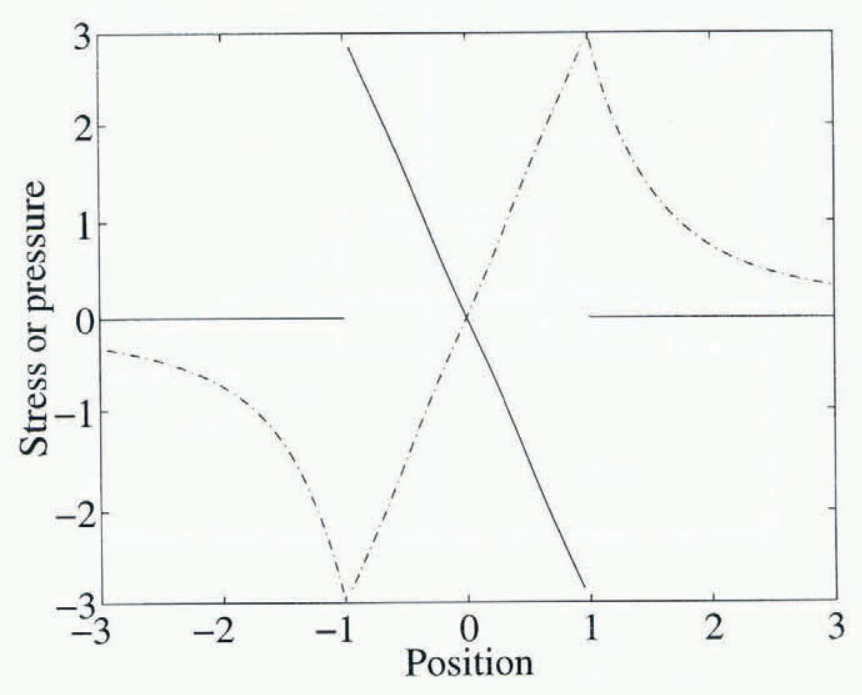

Fig. 3. The solutions for the radial stress $\sigma_{r r}$ (dash-dot line) given by Watts (1974) for flow around a sphere, and the water pressure in a thin water film (solid line) given by assuming the theory applies to a half-space. This normal traction is equal to the water pressure. Vertical axis is stress, horizontal axis $x$ is position in the direction of flow. The obstacle lies in $-1<x<1$. For $|x|>1$, the pressure is taken along a plane parallel to the direction of far-field flow which goes through the sphere centre (the bed plane), while for $|x|<1$ the stress and pressure are computed at the clast surface, i.e. along the surface $|r|=1$. Radial stress is continuous while normal traction is continuous.

"lift" generated on the clast is possible while global force balance is maintained over the clast and bed.

\subsection{Finite-element calculations}

We have suggested that the Watts solution is special owing to its symmetry, and we need to determine whether more general configurations show similar properties. We solve the Stokes equations

$$
\begin{aligned}
& \frac{\partial \tau_{x z}}{\partial z}+\frac{\partial \tau_{x x}}{\partial x}=\frac{\partial p}{\partial x} \\
& \frac{\partial \tau_{x z}}{\partial x}+\frac{\partial \tau_{z z}}{\partial z}=\frac{\partial p}{\partial z}
\end{aligned}
$$

along with a linear viscous relationship, where $(x, z)$ represents a two-dimensional Cartesian coordinate system with $z$ pointing upwards. Thermodynamic effects are not treated. Numerical (and therefore approximate) solutions are obtained by the finite-element discretisation procedure. We discretise using quadratic triangles with midside nodes where velocities are computed. Pressure as well as velocities are solved at the vertices of these triangles.

We investigate the flow over bumps (cf. Iken, 1981; Schweizer and Iken, 1992; Gudmundsson, 1994). At the base we assume perfect slip (zero tangential traction), and on the sides we use periodic-boundary conditions (so that the bump has an infinity of clones upstream and downstream). We apply the upper surface condition sufficiently far from the base that the vertical velocity 
component at the boundary can be assumed to be small. The boundary condition on this surface comprises a constant, unit, horizontal velocity and zero vertical velocity. A moving frame of reference can allow us to follow the sliding motion of a clast, since away from the clast/bump the bed is flat. Then, provided that we specify perfect slip over the bed, the solution also applies to a bump/clast travelling at arbitrary speed. We shall not specify physical constants, as we are interested in qualitative features of the flow.

Calculations were carried out for flow around a clast $c(x)$ specified by

$$
c(x)=\max \left[0,2 \sin \left(\frac{2 \pi x}{20}\right)-1\right] .
$$

Various grids were used, the densest of which is shown in Figure 4. These grids had varying numbers of computation nodes along the base: 17,33, 65 and 129. The normal traction at the ice-bed interface and from this the film

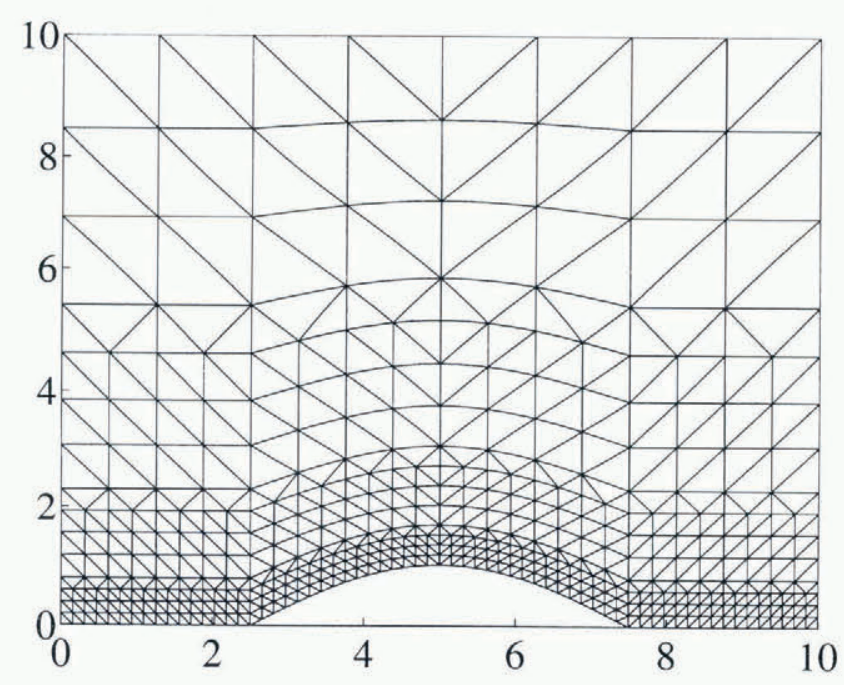

Fig. 4. Finite-element grid with 129 nodes along the botlom. Other grids, not shown here, used 65, 33 and 17 nodes along the base.

water pressure were computed. The results for various grids (Fig. 5) show convergent behaviour away from the leading and trailing edges but also evidence for discontinuities in water pressure at these edges. At clast edges the solution does not appear to converge. T. Jóhanneson (personal communication) has suggested that this non-convergence may be a manifestation of Gibbs' phenomena. An alternative way of viewing this is to note that discretisations of the problem, which smooth the slope discontinuity, are well posed, while the continuum representation is not, as it is not possible for a deforming fluid to sustain free surfaces meeting at angles other than a right-angle. The problem really arises because we have imposed the condition that the ice be conforming, whereas in general we would expect cavities to form.

Before proceeding on this point, we note that these solutions are symmetrical about the clast centre, meaning that the total normal force acting on the upper surface is zero and was found to be so to low error, owing to the

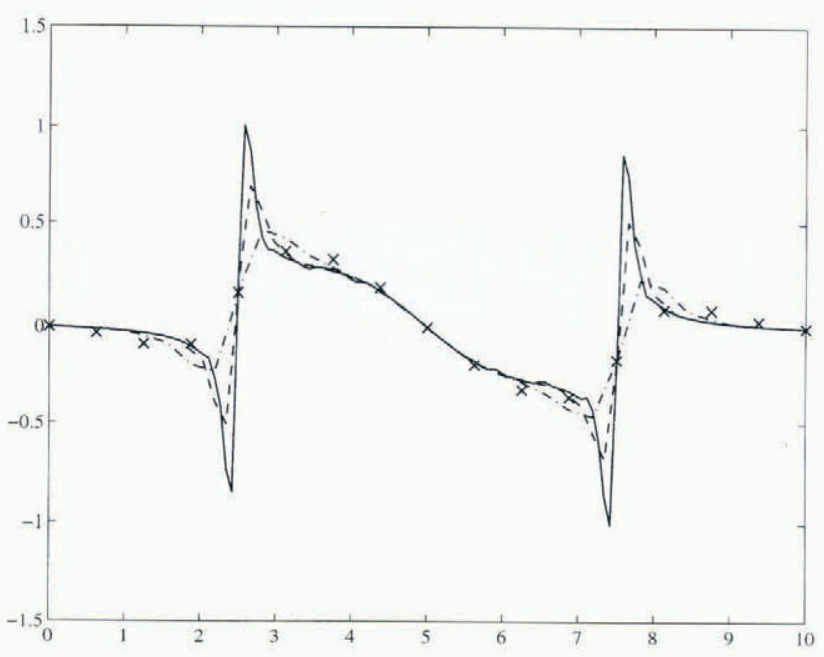

Fig. 5. Estimated normal traction (arbitrary units) plotted against horizontal position. The physical configuration of flow over a clast is shown in Figure 3, except that the different plots represent calculations with different computational grids. The unbroken line is for 129 nodes along the bottom, as in Figure 3; the dashed line is for 65 nodes along the bottom; the dash-dot line is for 33 nodes; the crosses are for 17 nodes.

good global conservation properties of the finite-element method. Various asymmetric bump configurations were used in similar calculations, and the ratio

$$
\frac{\int_{a}^{b} p_{\mathrm{w}} \mathrm{d} x}{\int_{a}^{b}\left|p_{\mathrm{w}}\right| \mathrm{d} x}
$$

computed. This was no longer exactly zero but still only of the order of a few per cent, and could not be said with any confidence to be different from zero. While steeper faces generate larger normal stresses, so increasing the magnitude of the force on the face, the vertical component of the normal vector is reduced, which reduces the net effect on the vertical force.

\section{THE THIN WATER FILM}

Regelation theory supposes that the thickness of the thin water film adjust so that the discharge of water $q_{\mathrm{w}}$ (determined by the melting and refreezing of ice) and the water-pressure gradient along the clast $\partial p_{\mathrm{w}} / \partial s$ (determined by the flow of ice around the clast) satisfy the laminar Poiseuille relation

$$
q_{\mathrm{w}}=-\frac{D^{3}}{12 \mu} \frac{\partial p_{\mathrm{w}}}{\partial s} .
$$

The problem of discontinuous water pressures has been considered by Weertman (1972) in his application of the Nye (1953) tunnel-closure theory. It is a problem that arises implicitly for all those who use the Röthlisberger (1972) theory of tunnel closure. In this application, the computed water pressure between the ice and the bed is different from the tunnel pressure (Fig. 6). Mollification of the discontinuous or singular behaviour is supposed to 


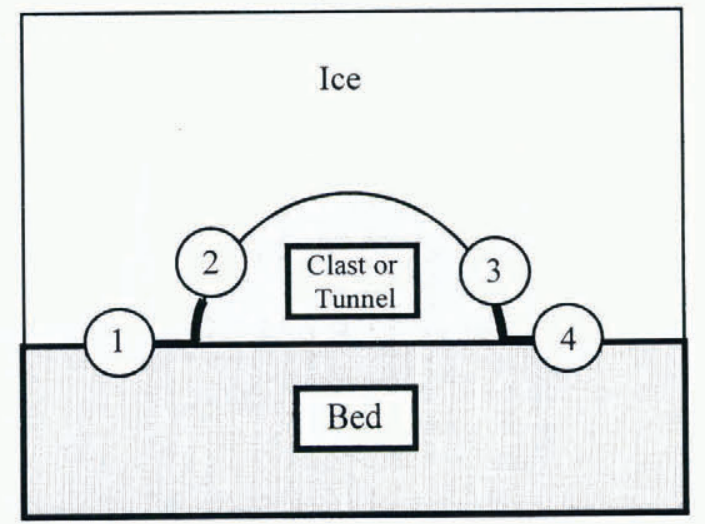

Fig. 6. The four possible water pressures associated with singular geometries. When this configuration represents a tunnel, the only water films are 1 and 4, and their pressure is different from the water pressure in the tunnel, except for a narrow boundary layer (Weertman, 1972). When this configuration represents ice flowing around the clast, films 1 and 2 belong to the leading edge, and 3 and 4 to the trailing edge; ice flow is from left to right. Film 1 is the leading-edge film abutting the bed; film 2 is the leadingedge film abutting the clast; film 3 is the trailing-edge pressure abutting the clast; film 4 is the trailing-edge pressure abutling the bed. In general, we expect the water pressures in these films to be effectively discontinuous at the corners. This leads to the formation of cavities.

occur by pinching-out of the water layer and consequent reduction of its transmissibility, allowing a large pressure gradient to exist. This can only happen when the water pressure in the film is higher than the pressure in the tunnel of cavity, as the opposite situation would simply lead to enlargement of the cavity. The high waterpressure gradients mimic the discontinuous behaviour and produce pressure-sealing. The Weertman theory has been criticised (e.g. Walder, 1982) because it appears to drive water away from tunnels. However, water may drain into tunnels through "Walderlets" (very small channels) while the thin water film described by Weertman mechanically supports nearly all of the ice. In short, criticisms of the Weertman theory as a description of drainage may be correct but do not affect my argument.

The solutions presented above for the flow of ice specified perfect slip as the boundary condition. This is correct if we assume that the pressure in the water film can take on arbitrary distributions, but, as the discussion above has shown, discontinuous water pressures are predicted in general. We hypothesise that the constriction of the water film creates a boundary layer in the flow of the ice, where the ice flow changes in order to accommodate the mechanical requirements of the waterpressure gradient. This boundary layer will have length scale parallel to the film comparable with the thickness of the thin water film. Away from the film the flow of ice is essentially undisturbed from the solution predicted by the perfect-slip condition. The pressure under the clast must be approximately constant, as this area is too transmissible to support a large pressure gradient.

We use this idea to consider qualitatively what happens at the leading and trailing edges of clasts. This is also illustrated in Figure 6. There are four pressures relevant to the problem, two associated with the leading edge, in the water film abutting the bed and the water film abutting the clast, and two associated with the trailing edge and its two associated water films. The water pressure underneath the clast is supposed to be constant. It could be less than all four of the water pressures generated by the ice flow over the clast, but only if there were a connection to an external drainage route through or along the bed.

Otherwise, we can consider the following cases, related to

1. The pressures at the trailing edge.

(a) The sub-clast pressure lies between the two trailing-edge pressures, being greater than the lower of the trailing-edge pressures, which is in the film abutting the clast lee face. A cavity opens, while the other, higher-pressure film, in the film abutting the bedrock, is sealed from the cavity by pinching-out of the water layer.

(b) If the sub-clast pressure is greater than both of the pressures associated with the trailing edge, the cavity grows until the extra bridging stress needed at the point of retouching exceeds the cavity pressure. This now corresponds to case 1 a.

2. The pressures at the leading edge.

(a) The sub-clast pressure is less than either of the leading-edge pressures. The ice is sucked under the clast (to a limited extent), and the leading-edge face and the bed face become sealed from each other and the clast by pinching-out of the water layer.

(b) The sub-clast pressure is greater than the lower of the two pressures. A leading-edge cavity is formed.

(c) The sub-clast pressure is greater than both leadingedge pressures. This can occur only if there is drainage into the sub-clast area from outside. The leading-edge cavity grows until increased bridging stresses cause the pressure in the film abutting the clast to be greater than the cavity pressure. This now corresponds to case $2 \mathrm{~b}$.

To illustrate these ideas, let us consider the flow of ice, initially at rest, around a particle lodged on the bed. The ice begins to flow, and a pressure difference is created along the clast. By the arguments above, we cannot expect the pressure to be less than the minimum trailingedge pressure, if the area under the clast is hydraulically isolated. The water pressure under the clast would then be a local minimum, and we would thus expect there to be flow of water into the area under the clast, raising the pressure above the minimum predicted by Watts-type theory. Under these conditions, a cavity will form, probably on the downstream side and possibly on the upstream side as well. Of course, if the minimum pressure predicted by Watts-type theory is less then the triplepoint pressure of water, a gas-filled cavity will form.

How does this phenomenon of discontinuous water pressure operate in three dimensions? We have seen from the Watts solution that the water pressure is discontinuous at the leading and trailing edges. As we move round the flanks of the clast, the discontinuity still exists but becomes smaller, until at the side there is no longer a 
discontinuity. Round the lee side of the clast the discontinuity reappears. Exactly the same arguments apply regarding the mollification of the discontinuous water pressure as in the two-dimensional case. Highpressure layers get pinched off by sealing, and the minimum sub-clast water pressure is determined by the lowest pressure in the system. Cavities can develop on the lee side.

This section is quite crucial, because it seems that we cannot compute the water pressure underneath the clast from fluid-dynamical considerations alone, which contradicts the implicit assumption made by Hallet (1979). As described above, this contradiction arises from the fact that Hallet does not consider the presence of the film abutting the bed, which connects the upstream and downstream sides of the clast. Instead, we have to consider the way the cavity is hydraulically connected to the rest of the glacier (Lliboutry, 1968; Walder, 1986; Fowler, 1987; Kamb, 1987).

A reviewer, B. Hallet, has rightly criticised the above model on the grounds that it ignores the thermodynamics of the problem. In particular, high pressure gradients imply high temperature gradients, and additional sources and sinks of water in the film. Since heat dissipation per unit area in the thin water film is given by $\mathbf{q} \cdot \nabla p_{\mathrm{w}}$, we can expect strong heating in the boundary layer. Moreover, if the pressure under the clast is lower than the ambient pressure, we should expect heat to flow out of the area, and freezing to occur underneath the clast which in fact is a problem for all hard-bed abrasion theories). All of these are valid points and may mean that sustained abrasion is not possible, as the clast could eventually get frozen into the ice. Acting against this particular mechanism is frictional heating between clast and bed. However, all that is needed for abrasion to occur is for the mechanical configurations described above to be realised temporarily, and this is quite plausible given that thermal time-scales are longer than mechanical time-scales, which can be regarded as being instantaneous.

\section{FORGE BALANCE AROUND CLASTS WITH CAVITIES}

If a clast is in contact with the bedrock, it must satisfy the Coulomb friction condition

$$
\left|F_{x}\right| \leq \eta\left|F_{z}\right|
$$

where $F_{x}$ is the force acting parallel to the bed, $F_{z}$ is the net normal contact force between clast and bed, and $\eta$ is the coefficient of friction.

There is an important problem relating to the presence of cavities around clasts, in that it has appeared theoretically quite difficult to generate normal and tangential forces on the clast which are consistent with the requirements of the Coulomb friction relationship (Boulton and others, 1979). This problem is reanalyzed with a hydraulic-jack model, and it is found that adjustment of the degree of cavitation can resolve the issue.

We carry out a semi-quantitative analysis as follows. We consider the clast to be the isosceles triangle sliding on its non-symmetric face, and we suppose that the water pressure declines linearly from leading to trailing edge. This configuration is illustrated in Figure 7. The clast profile is denoted by $z=c(x / X)$. Although this is a rather crude model, I believe that the analysis which follows is qualitatively correct; the decline in pressure seems to occur generally, while the faces of constant angle are devices to ease the computations below. The principal conclusion, which relates to how the ratio of horizontal to vertical forces changes with degree of cavitation, is qualitative.

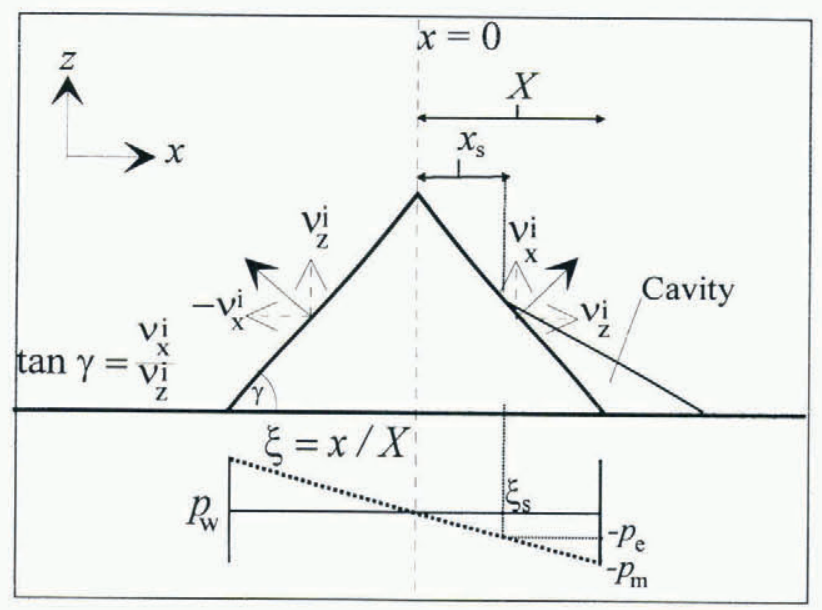

Fig. 7. The notation used in the clast force balance.

When a cavity exists, let us suppose that the film pressure is linear in $x$ from leading edge to point of separation on the trailing face or the trailing edge if no cavity exists and constant thereafter. Such linear dependence is found in Watts' solution. We express this pressure relationship as

$$
p_{\mathrm{w}}=\left\{\begin{array}{cl}
p_{\mathrm{i}}-p_{\mathrm{m}} \frac{x}{X}, & x<x_{\mathrm{s}} \\
p_{\mathrm{c}}, & x \geq x_{\mathrm{s}},
\end{array}\right.
$$

where $p_{\mathrm{m}}>0$ determines the water-pressure gradient and thus the water pressure at leading and trailing edges. The pressure in the cavity (if the cavity exists) is $p_{\mathrm{c}}$, and the water pressure underneath the clast is also $p_{\mathrm{c}}$. It is more convenient to consider the deviation of the water pressure from the ice pressure, and we thus write

$$
p_{\mathrm{w}}^{\prime}=p_{\mathrm{w}}-p_{\mathrm{i}},
$$

and in the cavity we have $p_{\mathrm{w}}^{\prime}=-p_{\mathrm{c}}=p_{\mathrm{c}}-p_{\mathrm{i}}$. These two relationships allow us to write

$$
p_{\mathrm{w}}^{\prime}= \begin{cases}-p_{\mathrm{m}} \frac{x}{X} & x<x_{\mathrm{s}} \\ -p_{\mathrm{e}} & x \geq x_{\mathrm{s}} .\end{cases}
$$

The point of separation is assumed to be given by

$$
\frac{x_{\mathrm{s}}}{X}=-\frac{p_{\mathrm{c}}-p_{\mathrm{i}}}{p_{\mathrm{m}}}=\frac{p_{e}}{p_{\mathrm{m}}},
$$

which is an assumption that after one has computed the position of separation, the presence of the cavity does not affect the pressure fields where the ice is still conforming 
to the clast. This construction amounts to a hydraulicjack-type theory (Röthlisberger and Iken, 1981). We define

$$
\xi=\frac{x}{X}
$$

and from Equation (4) note that in particular

$$
\xi_{\mathrm{s}}=\frac{p_{\mathrm{e}}}{p_{\mathrm{m}}}, \quad \frac{\mathrm{d} x_{\mathrm{s}}}{\mathrm{d} p_{\mathrm{e}}}=\frac{X}{p_{\mathrm{m}}} .
$$

Let us denote the normal vector

$$
\left(\nu_{x}, \nu_{z}\right)= \begin{cases}\left(-\nu_{x}^{\mathrm{i}}, \nu_{z}^{\mathrm{i}}\right), & \xi<0 \\ \left(\nu_{x}^{\mathrm{i}}, \nu_{z}^{\mathrm{i}}\right), & \xi>0,\end{cases}
$$

where $\nu_{x}^{\mathrm{i}}, \nu_{z}^{\mathrm{i}}$ are constants. By Cauchy's law the nonglaciostatic forces (i.e. subtracting the ice overburden) exerted by the water film on the upper surface of the clast are given by

$$
\left[\begin{array}{c}
F_{x}^{\mathrm{c}} \\
F_{z}^{\mathrm{c}}
\end{array}\right]=\left[\begin{array}{c}
-\int_{-S_{\mathrm{L}}}^{S_{\mathrm{R}}} p_{\mathrm{w}}^{\prime} \nu_{x} \mathrm{~d} s \\
-\int_{-S_{\mathrm{L}}}^{S_{\mathrm{R}}} p_{\mathrm{w}}^{\prime} \nu_{z} \mathrm{~d} s
\end{array}\right]
$$

where we recall $s$ is the distance along the clast surface. Use of the relationship

$$
\begin{aligned}
\mathrm{d} s & =\sqrt{1+\left(\frac{\partial c}{\partial x}\right)^{2}} \mathrm{~d} x=\sqrt{1+(\tan \gamma)^{2}} \mathrm{~d} x \\
& =\sqrt{1+\left(\frac{\nu_{x}}{\nu_{z}}\right)^{2}} \mathrm{~d} x
\end{aligned}
$$

where $\gamma$ is the angle subtended by the leading edge of the clast, allows us to write the non-glaciostatic forces as

$$
\left[\begin{array}{c}
F_{x}^{\mathrm{c}} \\
F_{z}^{\mathrm{c}}
\end{array}\right]=\left[\begin{array}{l}
-\int_{-X}^{X} p_{\mathrm{w}}^{\prime} \nu_{x} \sqrt{1+\left(\frac{\nu_{x}}{\nu_{z}}\right)^{2}} \mathrm{~d} x \\
-\int_{-X}^{X} p_{\mathrm{w}}^{\prime} \nu_{z} \sqrt{1+\left(\frac{\nu_{x}}{\nu_{z}}\right)^{2}} \mathrm{~d} x
\end{array}\right]
$$

which, upon using $|\vec{\nu}|=1$ allows us to write

$$
\left[\begin{array}{c}
F_{x}^{\mathrm{c}} \\
F_{z}^{\mathrm{c}}
\end{array}\right]=\frac{1}{\left|\nu_{z}\right|}\left[\begin{array}{c}
-\int_{-X}^{X} p_{\mathrm{w}}^{\prime} \nu_{x} \mathrm{~d} x \\
-\int_{-X}^{X} p_{\mathrm{w}}^{\prime} \nu_{z} \mathrm{~d} x
\end{array}\right]
$$

Note that we may also write

$$
F_{x}^{\mathrm{c}}=-\int_{-X}^{X} p_{\mathrm{w}}^{\prime} \tan \gamma \mathrm{d} x=-\int_{-X}^{X} p_{\mathrm{w}}^{\prime} \frac{\partial c}{\partial x} \mathrm{~d} x .
$$

From Equations (3) and (5) we can see that

$$
\begin{array}{ll}
p_{\mathrm{w}}^{\prime}=-p_{\mathrm{m}} \xi, & \xi \leq \xi_{\mathrm{s}}, \\
p_{\mathrm{w}}^{\prime}=-p_{\mathrm{e}}, & \xi>\xi_{\mathrm{s}},
\end{array}
$$

and

$$
\nu_{x}=-\operatorname{sgn}(\xi) \nu_{x}^{\mathrm{i}}, \quad \nu_{z}=-\nu_{z}^{\mathrm{i}} .
$$

The integrand in Equation $\left(7_{1}\right)$ can then be evaluated to be

$$
-p_{\mathrm{w}}^{\prime} \frac{\nu_{x}}{\nu_{z}}=p_{\mathrm{m}} \frac{\nu_{x}^{\mathrm{i}}}{\nu_{z}^{\mathrm{i}}}\left\{\begin{array}{ll}
|\xi|, & -1 \leq \xi \leq \xi_{\mathrm{s}} \\
\xi_{\mathrm{s}}, & \xi_{\mathrm{s}} \leq \xi \leq 1
\end{array}\right\} .
$$

With the integrands now defined, Equation (7) becomes

$$
\left[\begin{array}{c}
F_{x}^{\mathrm{c}} \\
F_{z}^{\mathrm{c}}
\end{array}\right]=\left[\begin{array}{c}
X\left(\frac{1}{\nu_{z}^{\mathrm{i}}} \int_{-1}^{\xi_{\mathrm{x}}} p_{\mathrm{m}}|\xi| \nu_{x}^{\mathrm{i}} \mathrm{d} \xi+p_{\mathrm{e}} c\left(\xi_{\mathrm{s}}\right)\right) \\
-X\left(\int_{-1}^{\xi_{\mathrm{s}}}-p_{\mathrm{m}} \xi \mathrm{d} \xi-p_{\mathrm{e}}\left(1-\xi_{\mathrm{s}}\right)\right)
\end{array}\right]
$$

and using Equation (6) to eliminate $p_{\mathrm{e}}$ yields

$$
\left[\begin{array}{c}
F_{x}^{\mathrm{c}} \\
F_{z}^{\mathrm{c}}
\end{array}\right]=\left[\begin{array}{c}
X p_{\mathrm{m}}\left(\int_{-1}^{\xi_{\mathrm{s}}} \frac{1}{\nu_{z}^{\mathrm{i}}}|\xi| \nu_{x}^{\mathrm{i}} \mathrm{d} \xi+\xi_{\mathrm{s}} c\left(\xi_{\mathrm{s}}\right)\right) \\
-X p_{\mathrm{m}}\left(\int_{-1}^{\xi_{\mathrm{s}}}-\xi \mathrm{d} \xi-\xi_{\mathrm{s}}\left(1-\xi_{\mathrm{s}}\right)\right)
\end{array}\right] .
$$

We note that

$$
c(\xi)=\epsilon X(1-|\xi|), \epsilon=\tan \gamma=\nu_{x}^{\mathrm{i}} / \nu_{z}^{\mathrm{i}} .
$$

Then, from Equation (8), we can write

$$
\left[\begin{array}{c}
F_{x}^{\mathrm{c}} \\
F_{z}^{\mathrm{c}}
\end{array}\right]=\left[\begin{array}{c}
\frac{\nu_{x}^{\mathrm{i}}}{\nu_{z}^{\mathrm{i}}} X p_{\mathrm{m}}\left(\int_{-1}^{\xi_{\mathrm{s}}}|\xi| \mathrm{d} \xi+\xi_{\mathrm{s}}\left(1-\left|\xi_{\mathrm{s}}\right|\right)\right) \\
-X p_{\mathrm{m}}\left(-\int_{-1}^{\xi_{\mathrm{s}}} \xi \mathrm{d} \xi-\xi_{\mathrm{s}}\left(1-\xi_{\mathrm{s}}\right)\right)
\end{array}\right]
$$

and computation of the integrals yields

$$
\left[\begin{array}{c}
F_{x}^{\mathrm{c}} \\
F_{z}^{\mathrm{c}}
\end{array}\right]=\left[\begin{array}{c}
\frac{1}{2} X \epsilon p_{\mathrm{m}}\left(1+\xi_{\mathrm{s}}\left(2-\left|\xi_{\mathrm{s}}\right|\right)\right) \\
-X p_{\mathrm{m}}\left(\frac{1}{2} \xi_{\mathrm{s}}^{2}+\frac{1}{2}-\xi_{\mathrm{s}}\right)
\end{array}\right]
$$

for the forces applied to the upper surface of the clast by the water film.

On the lower surface of the clast, the forces are given by

$$
\left[\begin{array}{c}
F_{x}^{\ell} \\
F_{z}^{\ell}
\end{array}\right]=\left[\begin{array}{c}
0 \\
-2 X p_{\mathrm{e}}
\end{array}\right]=\left[\begin{array}{c}
0 \\
-2 X p_{\mathrm{m}} \xi_{\mathrm{s}}
\end{array}\right],
$$

there being no $x$-direction forces owing to the assumed flat base. The total force $\mathbf{F}=\mathbf{F}^{c}+\mathbf{F}^{\ell}$, which is of course the clast-bed contact force, is found to be

$$
\left[\begin{array}{c}
F_{x} \\
F_{z}
\end{array}\right]=\left[\begin{array}{c}
\frac{1}{2} X \epsilon p_{\mathrm{m}}\left(1+\xi_{\mathrm{s}}\left(2-\left|\xi_{\mathrm{s}}\right|\right)\right) \\
-\frac{1}{2} X p_{\mathrm{m}}\left(\xi_{\mathrm{s}}+1\right)^{2}
\end{array}\right] .
$$

We define a function

$$
\Psi\left(\xi_{\mathrm{s}}\right)=\frac{1}{\epsilon} \frac{F_{x}}{\left|F_{z}\right|}=\frac{\left[1+\xi_{\mathrm{s}}\left(2-\left|\xi_{s}\right|\right)\right]}{\left(\xi_{\mathrm{s}}+1\right)^{2}} .
$$

The function $\Psi\left(\xi_{\mathrm{s}}\right)$ is shown in Figure 8 along with plots of $F_{x} / X \epsilon p_{\mathrm{m}},\left|F_{z}\right| / X p_{\mathrm{m}}$ and $\left|F_{z}^{\mathrm{c}}\right| / X p_{\mathrm{m}}$. For $\xi_{\mathrm{s}}=1$ (incipient cavitation) $\Psi\left(\xi_{\mathrm{s}}\right)=1 / 2$ while for $\xi_{\mathrm{s}}=0, \Psi\left(\xi_{\mathrm{s}}\right)=1$. For $\xi_{\mathrm{s}}<0, \Psi\left(\xi_{\mathrm{s}}\right)=1$, independent of the degree of cavitation. This case would correspond to positive effective pressure, which is unlikely to be physically sustainable, although it does not lead to to an upwards net force on the clast.

Figure 7 shows that we expect larger horizontal/ vertical force ratios as the cavity develops, although it should be remembered that this simple linearised model 


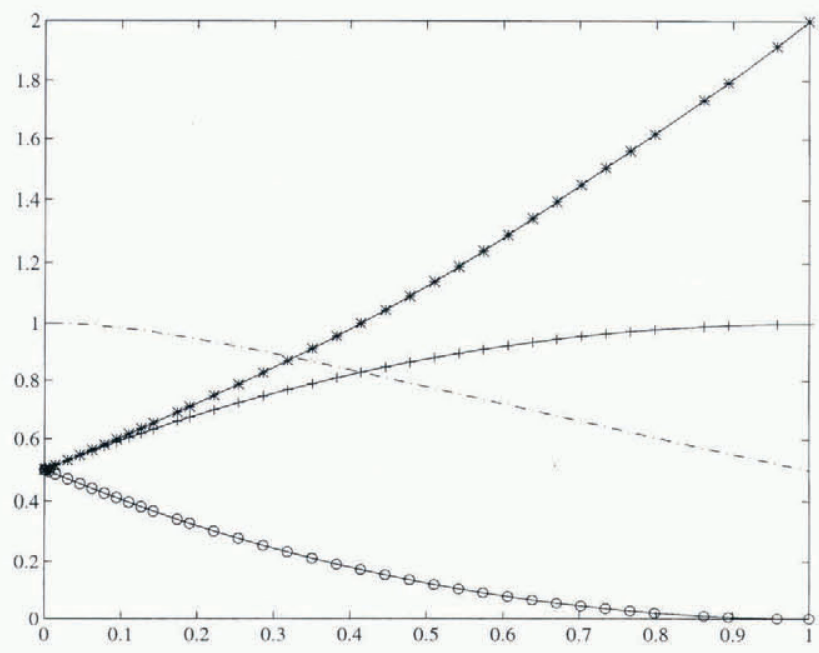

Fig. 8. Plots of the force ratio $\Psi\left(\xi_{\mathrm{s}}\right)$ (dash-dot); the horizontal force acting on the clast $F_{x}\left(\xi_{\mathrm{s}}\right)$ (crosses); the magnitude of the vertical force acting on the upper surface of the clast $\left|F_{z}\right|\left(\xi_{\mathrm{s}}\right)$ (asterisks); and the magnitude of the vertical force acting on the upper surface of the clast $\left|F_{z}^{\mathrm{c}}\right|\left(\xi_{\mathrm{s}}\right)$ (circles). This analysis ignores the effect of cavities developing at the leading edge.

of the stress distribution has ignored the possible development of stoss-side cavities near the leading edge. The Coulomb condition is now

$$
\Psi\left(\xi_{\mathrm{s}}\right) \leq \frac{\eta}{\epsilon}
$$

with the implication that, if $\Psi(0)=1>\eta / \epsilon$ while $\Psi(1)=\frac{1}{2} \leq \eta / \epsilon$, somewhere in between there will be a state of cavitation where the Coulomb condition is satisfied. This conclusion appears to be robust as it is simply based on continuity of the function $\Psi$, which is physically reasonable. Our conclusion is that if a clast is lodged, development of a cavity will lead to an increased horizontal/normal pressure ratio and the clast will begin to move provided that $1>\eta / \epsilon>\frac{1}{2}$. Clast-aspect ratio ("streamlining") is thus an important part of the problem. If we take $\eta=\tan 30^{\circ}$ this gives a the range of aspect ratios for mobile clasts between $30^{\circ}$ and $16^{\circ}$. Particles with too high an aspect ratio will, however, tend to get toppled.

The state of cavitation can be changed by altering $p_{\mathrm{e}}$, by drainage or imbibition or by the motion of the clast into zones of different ice pressure. For example, slow drainage of water into the cavity will cause the effective pressure to decrease, the cavity to grow and thus $\Psi$ to increase, possibly permitting clast motion. The state of cavitation will change if we change $p_{\mathrm{m}}$, which is determined by the relative velocity of ice and clast.

Another mechanism for changing the state of cavitation is to allow an increase in the relative velocity. This will increase the size of the cavity and increase $\Psi$, making conditions for movement of the clast more favourable. For a given cavity pressure and assuming that the clast is in motion, we can solve for the relative velocity of the clast and ice in much the same way as Boulton (1974) solved for this quantity. If the clast velocity is computed to be less than or equal to zero, then the clast is lodged.
Since the ratio of coefficient of friction to aspect ratio appears in the Coulomb condition, streamlined particles may not move (erode) on a rough bed, but if they are riding on a moving carpet of silt over polished bedrock, they may be able to move and erode. The implication is that smooth beds on the length scale of the clast and smaller) are preferentially eroded as they permit more clasts to move. There is a whole related question of which sediment fraction carries out the erosion (Riley, 1982; Cuffey and Alley, 1996; Hindmarsh, 1996); this is complicated by the fact that the presence of silt appears to inhibit abrasion.

This analysis is likely to be qualitatively correct for clasts which do not have a flat base. Consider the case where the leading and trailing edges lie a short distance above the bed. Underneath the clast, the water pressure will be constant, meaning that it exerts no resultant $x$ direction force on the clast. Ice may be present underneath the leading edge and (less likely) the trailing edge. There will still be distinct film pressures in the ice abutting the clast and the ice abutting the bed. Ice underneath the leading edge will tend to wedge the clast up, while deforming ice under the trailing edge should tend to pull the clast down. Since the latter is less likely, the net effect will be to reduce clast-bed contact forces, and conceivably that these could be sufficient to raise the clast off the bed. The horizontal force is less likely to be affected, as it is affected by the magnitude of the horizontal component of the normal vector but not the sign. Thus, clasts with curved bases are more likely to move, but some may not be able to stay in contact with the bed.

Bedward flow of ice should affect the cavity geometry. Intuitively, one would expect it to suppress cavitation. Combining this expectation with the prediction that decreased cavitation leads to a smaller horizontal/vertical force ratio suggests that bedward flow will increase the probability of a clast being lodged, in accordance with expectation.

I have criticised the Hallet model because of the singular geometries it implies, while having adopted such geometries myself. Is this as inconsistent as it appears? My argument is that singular geometries necessarily upset the assumption that the ice and bed are conformant, and this permits large pressure gradients which in turn permit the generation of large clast-bed contact forces. This is an additional mechanism, not requiring bedward flow, which may be able to generate larger bed-contact forces than the mechanism described by Hallet.

\section{CLAST-BED GONTACT FORGES}

Inspection of the force Equations (9) shows that the normal contact force $F_{z}$ depends on the tangential relative velocity through the parameter $p_{\mathrm{m}}$, which determines the pressure at the upstream face. This illustrates an important point, that in theories which involve cavitation, the normal contact forces depend on the tangential relative velocity, whereas in Hallet-type theories the normal contact forces depend on the bedward velocities. Clearly, theories which permit cavities to exist can potentially predict much larger contact stresses. The 
field evidence is equivocal. E. M. Morris (personal communication) states that from observations under Glacier d'Argentière, French Alps, clast velocities seemed to be very nearly the velocity of ice. Since the ice is sliding at hundreds of metres per year, it is not clear whether the relative velocity is tens of metres per year or tens of millimetres per year. The latter case would create contact stresses comparable with Hallet-type mechanisms involving bedward flow.

\section{CONGLUSIONS}

This paper argues that the pressure in the water film enveloping a clast cannot in general be determined by the flow of ice around the clast. The conforming flow of the ice around lubricated obstacles causes water-pressure discontinuities wherever there are breaks in slope in the clast-bed system. These breaks in slope are most likely to occur at the clast edges. Since such conforming flow is unlikely to be realised in practice, we expect cavities to form around the clast edges. These cavities are expected to be connected to the area under the clast. As in any subglacial cavities, water pressure is determined by connections to other parts of the bed. Even if large cavities do not grow, water pressure underneath the clast must be approximately constant.

This water pressure can be low enough to ensure clast-bed contact pressures higher than those generated by bedward flow, and will often be low enough to cause cavity formation on the lee side. It seems from hydraulicjack models that as the degree of cavitation increases, so too does the ratio of horizontal to vertical forces acting on the particle. The degree of cavitation is determined by the relative velocity of the ice and clast and the effective pressure of the clast cavity, and thus the degree of cavitation can adjust to satisfy the Coulomb condition.

As in any theory involving cavitation, the water pressure is not determined by the local flow, as it depends on the hydraulics of the bed. The theory in this paper can predict the maximum effective pressure realisable as the result of viscous flow around the clast and without sustained drainage of the water out of the sub-clast cavity. Drainage into the sub-clast cavity is potentially quite slow owing to the pinching-out of the water layers, and can actually start abrasion by increasing the ratio of horizontal to vertical force acting on the clast.

\section{ACKNOWLEDGEMENTS}

I would like to thank G.S. Boulton, I. Evans, E. M. Morris and J. F. Nye for instructive conversations over the past 15 years. B. Hallet, T. Jóhannesson and an anonymous reviewer all put substantial effort into improving this paper during the review phase. Part of this work was done while I was in receipt of a U.K. Natural Environment Research Council studentship at Durham University, supervised by I. Evans, whom I also thank for his advice, criticism and efforts. Some of these ideas were presented at a talk given by G.S. Boulton and R.C.A. Hindmarsh to the Institute of Physics in December 1985 .

\section{REFERENCES}

Boulton, G. S. 1974. Processes and patterns of glacial erosion. In Coates, D. R., ed. Glacial geomorphology). Binghamton, NY, State University of New York, 41-87.

Boulton, G.S., E. M. Morris, A. A. Armstrong and A. Thomas. 1979. Direct measurement of stress at the base of a glacier. \%. Glaciol., $2286,324$.

Cuffey, K. and R. Alley, 1996. Is crosion by deforming subglacial sediments significant? (Toward till continuity). Amn. Glaciol., 22 (sce paper in this volume).

Fowler. A. C. 1981. A theoretical treatment of the sliding of glaciers in the absence of cavitation. Philos. Trans, R. Soc, London, Ser. A, $2981445), 637 \quad 685$.

Fowler, A. C. 1987. Sliding with cavity formation. J. Glaciol., 33(115), $255-267$.

Gudmundsson, G.H. 1994. Glacier sliding over sinusoidal bed and the characteristics of creeping flow over bedrock undulations. Eidg. Tech. Hochschule, Zürich. Versuchsanst. Wasserbau, Hydrol. Glaziol. Mitt. 130.

Hallet, B. 1979. A theoretical model of glacial abrasion. J. Glaciol., $2389), 39-50$.

Hallet, B. 1981. Glacial abrasion and sliding: their dependence on the debris concentration in basal ice. Ann. Glaciol., 2, 23-28.

Hindmarsh, R.C.A. 1985. Modelling the development of glacial erosional landforms. (Ph.D. thesis, Durham University.)

Hindmarsh, R.C.A. 1996. Sliding of till over bedrock: scratching, polishing, comminution and kinematic-wave theory. Ann. Glaciol., 22 (see paper in this volume).

Iken, A. 1981. The effect of the subglacial water pressure on the sliding velocity of a glacier in an idealized numerical model. $\%$. Glaciol., $27(97), 407-421$.

Iverson, N.R. 1991. Morphology of glacial striae: implications for abrasion of glacier beds and fault surfaces. Geol. Soc. Am. Bull., $10310), 1308-1316$.

Kamb, B. 1970. Sliding motion of glaciers: theory and observation. Rev. Geophys. Space Plys., 8(4), 673-728.

Kamb, B. 1987. Glacier surge mechanism based on linked-cavity configuration of the basal water conduit system. 7. Geophys. Res., 92 B9), 9083-9100.

Lliboutry, L. 1968. General theory of subglacial cavitation and sliding of temperate glaciers. J. Glaciol., 7 49), 21-58.

Nye, J.F. 1953. The flow law of ice from measurements in glacier tunnels, laboratory experiments and the Jungfraufirn borehole experiment. Proc. R. Soc. London, Ser. A, 219 (1139), 477489.

Nye, J.F. 1969. A calculation on the sliding of ice over a wavy surface using a Newtonian viscous approximation. Proc. R. Soc. London, Ser. A, $311(1506), 445-467$.

Riley, N. W. 1982. Rock wear by sliding ice. Ph.D. thesis, University of Newcastle upon Tyne.)

Robin, G. de Q. 1976. Is the basal ice of a temperate glacier at the pressure melting point? J. Glaciol., 16 74), $183-196$.

Röthlisberger, H. 1972. Water pressure in intra- and subglacial channels, f. Glaciol., $11(62), 177-203$.

Röthlisberger, H. and A. Iken. 1981. Plucking as an effect of waterpressure variations at the glacier bed. Amn. Glaciol., 2, 57-62.

Schweizer. J. and A. Iken. 1992. The role of bed separation and friction in sliding over an undeformable bed. J. Glaciol., 38 128), 77-92.

Walder, J.S. 1982. Stability of sheet flow of water beneath temperate glaciers and implications for glacier surging. J. Glaciol., 28 (99), 273293.

Walder, J. S. 1986. Hydraulics of subglacial cavities, J. Glaciol., 32(112), 439445.

Watts, P.A. 1974. Inclusions in ice. (Ph.D. thesis, University of Bristol.)

Weertman, J. 1972. General theory of water flow at the base of a glacier or ice sheet. Rev. Geophys. Space Plyss., 10 (1), 287-333. 